\title{
A Well-Conditioned Combined Field Integral Equation Based on Quasi-Helmholtz Projectors
}

\author{
Francesco P. Andriulli* $\quad$ Ignace Bogaert ${ }^{\dagger} \quad$ Kristof Cools $^{\ddagger} \quad$ Eric Michielssen ${ }^{\S}$
}

\begin{abstract}
We introduce a novel combined field integral equation that does not suffer from internal resonances and solves several drawbacks of existing resonance-free formulations. The new equation is obtained by combining a regularized electric type operator with a new magnetic type operator that exhibits uniform frequency scaling when acting on, or being tested within, the harmonic Helmholtz subspace for surface currents. With an appropriate use of quasi-Helmholtz projectors, the equation is stable for arbitrarily low-frequencies. Numerical results confirm the theoretical developments and show the effectiveness of the scheme.
\end{abstract}

\section{INTRODUCTION}

All known integral equation techniques for simulating scattering and radiation from arbitrarily shaped, perfect electrically conducting objects suffer from one or more of the following shortcomings: (i) they give rise to ill-conditioned systems when the frequency is low (ii) and/or when the discretization density is high; (iii) their applicability is limited to the quasi-static regime; (iv) they require a search for global topological loops; (v) they suffer from numerical cancelations in the solution when the frequency is very low. A recent paper [1] presented a new integral operator of the electric type that does not suffer from any of the above drawbacks.

When the scatterer under consideration is closed, integral operators of the electric and magnetic type suffer from interior resonances, i.e. null-spaces for wavenumbers that correspond to a resonance of an interior problem. The presence of these resonances often negatively impacts the accuracy and solution time of solvers leveraging these operators. When standard electric and magnetic field operators are used, this problem can be remedied by using a combined field operator, viz. a linear combination of the electric and magnetic field operator that is provably resonance-free. Unfortunately, standard combined field operators inherit all the drawbacks (i)-(v).

This contribution extends the recently developed

* Microwave Departement, École Nationale Supérieure des Télécommunications de Bretagne (TELECOM Bretagne), email:francesco.andriulli@telecom-bretagne.eu

$\dagger$ University of Ghent, e-mail:ibogaert@ugent.be

$¥$ University of Nottingham,

e-mail:kristof . cools@nottingham.ac.uk

$\S$ University of Michigan, e-mail:emichiel@umich.edu electric type operator immune from (i)-(v) by developing a new magnetic type operator that can be used to construct a combined field operator that does not suffer from (i)-(v) and is immune from interior resonances, i.e. that is uniquely solvable for all frequencies. The new formulation is obtained starting from a Helmholtz decomposition of two discretizations of the electric field integral operator and from a suitably symmetrized mixed discretization of the magnetic field integral operator obtained by using RWGs and dual bases functions, respectively. The new decomposition does not leverage Loop and Star/Tree basis functions; rather, it employs projectors that derive from them and does not require the explicit detection of global topological loops. The theoretical developments will be corroborated by numerical results, confirming the effectiveness of the newly developed method.

\section{BACKGROUND AND FORMULA- TION}

Consider the surface $\Gamma$ of an orientable PEC object residing in a space of permittivity $\epsilon$ and permeability $\mu$. Denote with $\hat{\boldsymbol{n}}_{\boldsymbol{r}}$ its outward pointing unit normal at $\boldsymbol{r}$. Denote with $\Omega^{+}$and $\Omega^{-}$the exterior and interior regions of $\Gamma$, respectively. An incident electromagnetic field $\left(\boldsymbol{E}^{i}(\boldsymbol{r}), \boldsymbol{H}^{i}(\boldsymbol{r})\right)$ impinges on $\Gamma$, inducing a surface current density $\boldsymbol{J}(\boldsymbol{r})$. The current density $\boldsymbol{J}(\boldsymbol{r})$ can be retrieved by solving the Electric Field Integral Equation (EFIE)

$$
\mathcal{T}_{k}(\boldsymbol{J})=-\hat{\boldsymbol{n}}_{\boldsymbol{r}} \times \boldsymbol{E}^{i}
$$

where $\mathcal{T}_{k}(\boldsymbol{J})=i k \mathcal{T}_{s}(\boldsymbol{J})+\frac{1}{i k} \mathcal{T}_{h}(\boldsymbol{J})$ with

$$
\begin{aligned}
& \mathcal{T}_{s}(\boldsymbol{J})=\hat{\boldsymbol{n}}_{\boldsymbol{r}} \times \int_{\Gamma} \frac{e^{i k\left|\boldsymbol{r}-\boldsymbol{r}^{\prime}\right|}}{4 \pi\left|\boldsymbol{r}-\boldsymbol{r}^{\prime}\right|} \boldsymbol{J}\left(\boldsymbol{r}^{\prime}\right) \mathrm{d} \boldsymbol{r}^{\prime}, \\
& \mathcal{T}_{h}(\boldsymbol{J})=-\hat{\boldsymbol{n}}_{\boldsymbol{r}} \times \nabla \int_{\Gamma} \frac{e^{i k\left|\boldsymbol{r}-\boldsymbol{r}^{\prime}\right|}}{4 \pi\left|\boldsymbol{r}-\boldsymbol{r}^{\prime}\right|} \nabla_{s} \cdot \boldsymbol{J}\left(\boldsymbol{r}^{\prime}\right) \mathrm{d} \boldsymbol{r}^{\prime},
\end{aligned}
$$

and $k=2 \pi / \lambda=\omega \sqrt{\epsilon \mu}$. Alternatively, if $\Gamma$ is closed, $\boldsymbol{J}(\boldsymbol{r})$ can be retrieved by solving the Magnetic Field Integral Equation (MFIE)

$$
\left(\frac{\mathcal{I}}{2}+\mathcal{K}_{k}\right)(\boldsymbol{J})=\eta\left(\hat{\boldsymbol{n}}_{\boldsymbol{r}} \times \boldsymbol{H}^{i}\right)
$$


where

$$
\mathcal{K}_{k}(\boldsymbol{J})=-\hat{\boldsymbol{n}}_{\boldsymbol{r}} \times \int_{\Gamma} \nabla \frac{e^{i k\left|\boldsymbol{r}-\boldsymbol{r}^{\prime}\right|}}{4 \pi\left|\boldsymbol{r}-\boldsymbol{r}^{\prime}\right|} \times \boldsymbol{J}\left(\boldsymbol{r}^{\prime}\right) \mathrm{d} \boldsymbol{r}^{\prime}
$$

and $\eta=\sqrt{\mu / \epsilon}$. Note that with these definitions the current $\boldsymbol{J}(\boldsymbol{r})$ represents the jump over $\Gamma$ of the total magnetic field multiplied by the medium characteristic impedance.

When the surface $\Gamma$ is closed and when $k$ corresponds to an interior resonance, i.e. to an eigenvalue of the Laplacian in $\Omega^{-}$, both the EFIE and MFIE are not solvable [2]. When $k$ approaches such a resonant value, the linear systems arizing from the discretization of the EFIE and MFIE become almost singular and difficult to solve. A similar problem plagues the Calderón preconditioned EFIE. A classical solution to this problem leverages the Combined Field Integral Equation (CFIE) [3], viz. a linear combination of EFIE and MFIE

$$
\begin{aligned}
\alpha \mathcal{T}_{k}(\boldsymbol{J})+\left(\frac{\mathcal{I}}{2}\right. & \left.+\mathcal{K}_{k}\right)(\boldsymbol{J})= \\
& =-\alpha \hat{\boldsymbol{n}}_{\boldsymbol{r}} \times \boldsymbol{E}^{i}+\eta\left(\hat{\boldsymbol{n}}_{\boldsymbol{r}} \times \boldsymbol{H}^{i}\right)
\end{aligned}
$$

where $\alpha \neq 0$ and real. The CFIE can be proven to be uniquely solvable for all values of $k$. On the other hand, the presence of the EFIE operator $\mathcal{T}$ results in ill-conditioning that, although milder than the ill-conditioning of the EFIE, still renders the equation difficult to solve in many real case scenarios. One could attempt to obtain a resonance-free and well-conditioned equation by linearly combining the MFIE and the Calderón preconditioned EFIE as

$$
\begin{aligned}
\alpha \mathcal{T}_{k}^{2}(\boldsymbol{J}) & +\left(\frac{\mathcal{I}}{2}+\mathcal{K}_{k}\right)(\boldsymbol{J})= \\
& =-\alpha \mathcal{T}_{k}\left(\hat{\boldsymbol{n}}_{\boldsymbol{r}} \times \boldsymbol{E}^{i}\right)+\eta\left(\hat{\boldsymbol{n}}_{\boldsymbol{r}} \times \boldsymbol{H}^{i}\right) .
\end{aligned}
$$

Unfortunately this choice does not lead to a resonance-free equation since

$$
\mathcal{T}_{k}^{2}=-\frac{\mathcal{I}}{2}+\mathcal{K}_{k}^{2}=-\left(\frac{\mathcal{I}}{2}-\mathcal{K}_{k}\right)\left(\frac{\mathcal{I}}{2}+\mathcal{K}_{k}\right)
$$

i.e. the null space of $\mathcal{T}_{k}^{2}$ contains that of $\left(\frac{\mathcal{I}}{2}+\mathcal{K}_{k}\right)$ and so does (7). A way to solve this problem is to precondition the EFIE operator $\mathcal{T}$ not with the operator $\mathcal{T}$ itself, but with its localized counterpart. This localization can be obtained either by a space windowing the Green's function [4] or by using, in the leftmost operator $\mathcal{T}$, a purely complex wavenumber, as was proposed in [5], obtaining

$$
\mathcal{C}(\boldsymbol{J})=-\alpha \mathcal{T}_{i k}\left(\hat{\boldsymbol{n}}_{\boldsymbol{r}} \times \boldsymbol{E}^{i}\right)+\eta\left(\hat{\boldsymbol{n}}_{\boldsymbol{r}} \times \boldsymbol{H}^{i}\right)
$$

where

$$
\mathcal{C}(\boldsymbol{J})=\alpha \mathcal{T}_{i k} \mathcal{T}_{k}(\boldsymbol{J})+\left(\frac{\mathcal{I}}{2}+\mathcal{K}_{k}\right)(\boldsymbol{J}) .
$$

Although (9) is immune from both low-frequency and dense discretization breakdown, it can be shown to suffer from very low-frequency solution cancelations and to have a static null-space whenever the geometry under consideration contains handles or holes (i.e. for non-simply connected geometries). The reader is referred to [1] for a detailed discussion of these phenomena. Recently, an electric operator that is immune from the above mentioned problems has been proposed [1]. This operator is obtained by using Helmholtz projectors that properly alter the low frequency behavior of the standard Calderon EFIE. The resulting equation has been shown to be stable till very low frequency even for non-simply connected geometries. Being of electric type, the operator defined in [1] still suffers from internal resonances and hence needs to be included in a suitable combined field formulation. This is the objective of this contribution.

The reader should note that a key property of the EFIE operator, fundamental for realizing the properties obtained in [1] is that, if we consider the harmonic subspace of the Helmholtz decomposition of the surface current, the EFIE operator has the same frequency scaling when being applied to or being tested with any element of the harmonic subspace. This key property, unfortunately, does not hold for MFIE operator. This is the reason why it is not possible to obtain an effective combined field formulations by simply using the electric type operator in [1] in an equation like (9). Instead, a new magnetic type operator should be defined that scales in a uniform way when acting on or tested with the harmonic Helmholtz subspaces. This will be the subject of next session where a new combined field formulation will be proposed together with the proof of its resonance-free behavior.

\section{A NEW COMBINED FIELD EQUA- TION}

The new combined field equation we propose reads

$$
\begin{gathered}
\left(\eta^{2}\left(\frac{\mathcal{I}}{2}-\mathcal{K}_{i k}\right)\left(\frac{\mathcal{I}}{2}+\mathcal{K}_{k}\right)(k)+\mathcal{T}_{i k} \mathcal{T}_{k}\right)(\boldsymbol{J})= \\
\left(\frac{\mathcal{I}}{2}-\mathcal{K}_{i k}\right)\left(\hat{\boldsymbol{n}}_{\boldsymbol{r}} \times \boldsymbol{H}\right)+\mathcal{T}_{i k}\left(\hat{\boldsymbol{n}}_{\boldsymbol{r}} \times \boldsymbol{E}\right)
\end{gathered}
$$

We now prove that then the operator

$$
\left(\left(\frac{\mathcal{I}}{2}-\mathcal{K}_{i k}\right)\left(\frac{\mathcal{I}}{2}+\mathcal{K}_{k}\right)(k)+\mathcal{T}_{i k} \mathcal{T}_{k}\right)
$$


is always invertible $\forall k$. Given that $\left(\frac{\mathcal{I}}{2}-\mathcal{K}\right)(i k)$ is always invertible, we will study the operator

$$
\left(\frac{\mathcal{I}}{2}+\mathcal{K}_{k}\right)+\left(\left(\frac{\mathcal{I}}{2}-\mathcal{K}_{i k}\right)\right)^{-1} \mathcal{T}_{i k} \mathcal{T}_{k}
$$

The following commutation

$$
\mathcal{T}^{-1} \mathcal{K}+\mathcal{K} \mathcal{T}^{-1}=0
$$

is a straightforward consequence of the second Calderón identity $\mathcal{T}^{-1} \mathcal{K}=\mathcal{T}^{-1} \mathcal{K} \mathcal{T} \mathcal{T}^{-1}=$ $-\mathcal{T}^{-1} \mathcal{T} \mathcal{K} \mathcal{T}^{-1}=-\mathcal{K} \mathcal{T}^{-1}$. Thus, defining

$$
\mathcal{A}=\left(\left(\frac{\mathcal{I}}{2}-\mathcal{K}_{i k}\right)\right)^{-1} \mathcal{T}_{i k}
$$

we obtain

$$
\begin{aligned}
& \left.\left(\hat{\boldsymbol{n}}_{\boldsymbol{r}} \times \mathcal{A}\right)^{T}=\left(\hat{\boldsymbol{n}}_{\boldsymbol{r}} \times\left(\left(\frac{\mathcal{I}}{2}-\mathcal{K}_{i k}\right)\right)^{-1} \mathcal{T}_{i k}\right)\right)^{T} \\
& =\left(\hat{\boldsymbol{n}}_{\boldsymbol{r}} \times\left(\mathcal{T}_{i k}^{-1}\left(\frac{\mathcal{I}}{2}-\mathcal{K}_{i k}\right)\right)^{-1}\right)^{T} \\
& \left.=\left(\hat{\boldsymbol{n}}_{\boldsymbol{r}} \times\left(\left(\frac{\mathcal{I}}{2}+\mathcal{K}_{i k}\right)\right) \mathcal{T}_{i k}^{-1}\right)^{-1}\right)^{T} \\
& =\left(\hat{\boldsymbol{n}}_{\boldsymbol{r}} \times \mathcal{T}_{i k}\left(\left(\frac{\mathcal{I}}{2}+\mathcal{K}_{i k}\right)\right)^{-1}\right)^{T} \\
& =\left(\left(\left(\frac{\mathcal{I}}{2}+\mathcal{K}_{i k}\right)\right)^{-1}\right)^{T} \hat{\boldsymbol{n}}_{\boldsymbol{r}} \times \mathcal{T}_{i k} \\
& =-\hat{\boldsymbol{n}}_{\boldsymbol{r}} \times\left(\left(\frac{\mathcal{I}}{2}-\mathcal{K}_{i k}\right)\right)^{-1} \hat{\boldsymbol{n}}_{\boldsymbol{r}} \times \hat{\boldsymbol{n}}_{\boldsymbol{r}} \times \mathcal{T}_{i k} \\
& =\hat{\boldsymbol{n}}_{\boldsymbol{r}} \times\left(\left(\frac{\mathcal{I}}{2}-\mathcal{K}_{i k}\right)\right)^{-1} \mathcal{T}_{i k} \\
& =\hat{\boldsymbol{n}}_{\boldsymbol{r}} \times \mathcal{A} .
\end{aligned}
$$

Finally, given that $\hat{\boldsymbol{n}}_{\boldsymbol{r}} \times \mathcal{A}=\hat{\boldsymbol{n}}_{\boldsymbol{r}} \times$ $\left(\left(\frac{\mathcal{I}}{2}-\mathcal{K}_{i k}\right)\right)^{-1} \mathcal{T}_{i k} \quad$ is a real operator, the symmetry implies it being hermitian, so that $\boldsymbol{x}^{\dagger}\left(\hat{\boldsymbol{n}}_{\boldsymbol{r}} \times\left(\left(\frac{\mathcal{I}}{2}-\mathcal{K}_{i k}\right)(i k)\right)^{-1} \mathcal{T}_{i k}\right) \boldsymbol{x}$ is real and nonzero. By leveraging a straightforward extension of Theorem 3.1 in [6], it follows that

$$
\left(\left(\frac{\mathcal{I}}{2}-\mathcal{K}_{i k}\right)\left(\frac{\mathcal{I}}{2}+\mathcal{K}_{k}\right)+\mathcal{T}_{i k} \mathcal{T}_{k}\right)
$$

is always invertible.

\section{DISCRETIZATION STRATEGY}

The new operator will be discretized by adopting a mixed-discretization strategy $[7,8]$ where the magnetic operators are tested with Buffa-Christiansen
(BC) basis functions. For the sake of brevity we consider a discretization for the case of simply connected structures; minor modifications are required for the non-simply connected case. The discretization reads:

$$
\begin{aligned}
& \left(\eta^{2} \mathbb{M}\left(\frac{\overline{\overline{\mathbf{G}}}_{m i x}^{T}}{2}-\overline{\overline{\mathbf{K}}}_{m i x}^{i k}\right)\left(\overline{\overline{\mathbf{G}}}_{m i x}^{T}\right)^{-1} .\right. \\
& \cdot\left(\frac{\overline{\overline{\mathbf{G}}}_{m i x}^{T}}{2}+\overline{\overline{\mathbf{K}}}_{m i x}^{k}\right) \overline{\overline{\mathbf{M}}} \\
& \left.+\mathbb{M} \overline{\overline{\mathbf{T}}}_{B C}^{i k} \mathbb{M} \overline{\overline{\mathbf{G}}}_{m i x}^{-1} \overline{\overline{\mathbf{M}}} \overline{\overline{\mathbf{T}}}_{R W G}^{k} \mathbf{\overline { \mathbf { M } }}\right) \overline{\mathbf{I}}= \\
& \eta^{2} \mathbb{M}\left(\frac{\overline{\mathbf{G}}_{m i x}^{T}}{2}-\overline{\overline{\mathbf{K}}}_{m i x}^{i k}\right)\left(\overline{\overline{\mathbf{G}}}_{m i x}^{T}\right)^{-1} \overline{\mathbf{V}}_{H} \\
& +\mathbb{M} \overline{\overline{\mathbf{T}}}_{B C}^{i k} \mathbb{M} \overline{\overline{\mathbf{G}}}_{m i x}^{-1} \overline{\overline{\mathbf{M}}} \overline{\mathbf{V}}
\end{aligned}
$$

where $\left(\overline{\overline{\mathbf{T}}}_{R W G}^{k}\right)_{i, j}=\left\langle\hat{\boldsymbol{n}}_{\boldsymbol{r}} \times \boldsymbol{f}_{i}, \mathcal{T}_{k}\left(\boldsymbol{f}_{j}\right)\right\rangle$ and $\left(\overline{\overline{\mathbf{T}}}_{B C}^{k}\right)_{i, j}=\left\langle\hat{\boldsymbol{n}}_{\boldsymbol{r}} \times \boldsymbol{f}_{i}^{B C}, \mathcal{T}_{k}\left(\boldsymbol{f}_{j}^{B C}\right)\right\rangle$ are the EFIE operators discretized with standard RWG $f$ [9] and BC basis functions $\boldsymbol{f}^{B C}$ [10] respectively, $\left(\overline{\mathbf{K}}_{m i x}^{k}\right)_{i, j}=\left\langle\hat{\boldsymbol{n}}_{\boldsymbol{r}} \times \boldsymbol{f}_{i}^{B C}, \mathcal{K}_{k}\left(\boldsymbol{f}_{j}\right)\right\rangle$ is the mixed discretized MFIE operator, $\left(\overline{\overline{\mathbf{G}}}_{m i x}\right)_{i, j}=$ $\left\langle\hat{\boldsymbol{n}}_{\boldsymbol{r}} \times \boldsymbol{f}_{i}, \boldsymbol{f}_{j}^{B C}\right\rangle$ is the mixed Gram matrix, $(\overline{\mathbf{V}})_{i}=-\left\langle\hat{\boldsymbol{n}}_{\boldsymbol{r}} \times \boldsymbol{f}_{i}, \hat{\boldsymbol{n}}_{\boldsymbol{r}} \times \boldsymbol{E}^{i}\right\rangle$ and $\left(\overline{\mathbf{V}}_{H}\right)_{i}=$ $-\left\langle\hat{\boldsymbol{n}}_{\boldsymbol{r}} \times \boldsymbol{f}_{i}^{B C}, \eta\left(\hat{\boldsymbol{n}}_{\boldsymbol{r}} \times \boldsymbol{H}^{i}\right)\right\rangle$ are the electric and magnetic right hand sides respectively, and $(\overline{\mathbf{I}})_{j}=$ $I_{j}$ is the unknown current vector such that $\boldsymbol{J}(\boldsymbol{r}) \approx$ $\sum_{n=1}^{N} I_{n} \boldsymbol{f}_{n}(\boldsymbol{r})$. Finally, the definition of the projectors $\mathbb{M}$ and $\overline{\mathbf{M}}$ is omitted here for space limitations, but it can be found in [1] for low frequency simulations. They are set equal to the identity, instead, for high frequency ones.

\section{NUMERICAL RESULTS}

The numerical tests involve a sphere of unit radius that is excited by a plane wave. The fact that the new equation is immune from the very low frequency current cancelation is confirmed by Fig. 1(a) which show the far field calculated using (13) at $10^{-40} \mathrm{~Hz}$. From Fig. 1(a) it is clear that although a standard Calderón equation can provide a stable solution till relatively low frequencies, the new equation is immune from the very low frequency current cancelation and provides stable solutions even when the frequency is arbitrarily low. The resonance free behavior of the new equation is tested in Fig. 1(b) where the new formulation clearly shows to be resonance-free.

\section{Acknowledgments}

This work has been partially funded by the ANR (Agence Nationale de la Recherche) under the 


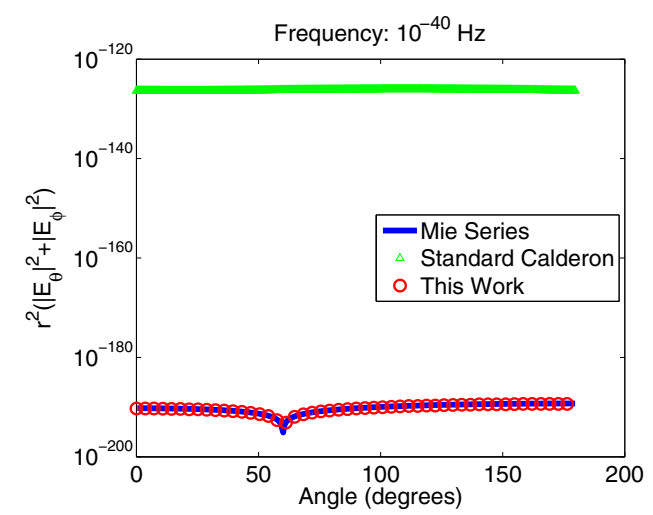

(a) Sphere: far field calculated when the frequency equals $10^{-40} \mathrm{~Hz}$.

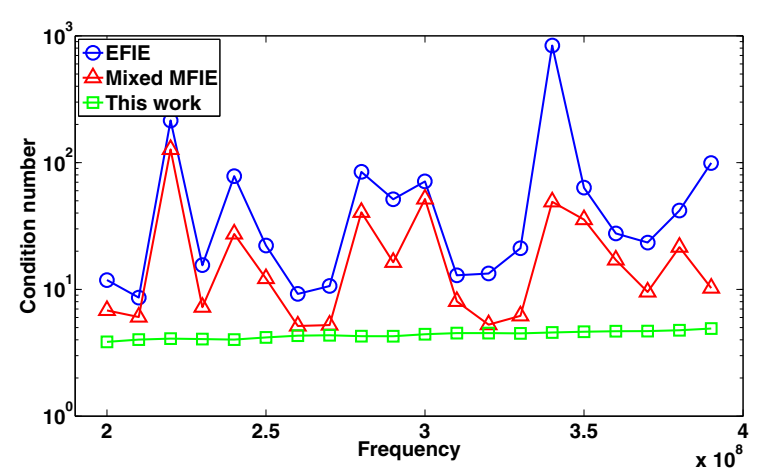

(b) Sphere: condition number as a function of frequency.

Figure 1: Numerical results

project FASTEEG-ANR-12-JS09-0010. One of the authors also thank GENCI (Grand Equipement National de Calcul Intensif) for providing the necessary computing facilities for this work.

\section{References}

[1] F. P. Andriulli, K. Cools, I. Bogaert, and E. Michielssen, "On a well-conditioned electric field integral operator for multiply connected geometries," IEEE Trans. Antennas Propagation, vol. 61, no. 4, pp. 2077-2087, 2013.

[2] J.-C. Nedéléc, Acoustic and Electromagnetic Equations. Springer, 2000.

[3] J. R. Mautz and R. F. Harrington, "H-field, Efield, and combined field solution for conducting bodies of revolution," Archiv. f. Elektronik u. Ubertragungstechnik, vol. 32, pp. 159-164, 1978.

[4] R. J. Adams, "Physical and analytical properties of a stabilized electric field integral equation," IEEE Trans. Antennas Propagation, vol. 52, no. 2, pp. 362-372, Feb. 2004.

[5] H. Contopanagos, B. Dembart, M. Epton, J. Ottusch, V. Rokhlin, J. Visher, and S. M. Wandzura, "Well-Conditioned Boundary Integral Equations for Three-Dimensional Electromagnetic Scattering," IEEE Trans. Antennas Propagation, vol. 50, no. 12, pp. 1824-1930, Dec. 2002.

[6] O. Bruno, T. Elling, R. Paffenroth, and C. Turc, "Electromagnetic Integral Equations Requiring Small Numbers of Krylov-subspace Iterations," Journal of Computational Physics, vol. 228, pp. 6169-6183, 2009.
[7] K. Cools, F. P. Andriulli, D. D. Zutter, and E. Michielssen, "Accurate and conforming mixed discretization of the mfie," IEEE Antennas and Wireless Propagation Letters, vol. 10, pp. 528-531, 2011.

[8] I. Bogaert, K. Cools, F. Andriulli, and D. De Zutter, "Low frequency scaling of the mixed mfie for scatterers with a non-simply connected surface," in International Conference on Electromagnetics in Advanced Applications (ICEAA), 2011, sept. 2011, pp. 951 954.

[9] S. M. Rao, D. R. Wilton, and A. W. Glisson, "Elettromagnetic scattering by surfaces of arbitrary shape," IEEE Trans. Antennas Propagation, vol. AP-30, no. 3, pp. 409-418, May 1982.

[10] A. Buffa and S. H. Christiansen, "A dual finite element complex on the barycentric refinement," Math. Comp., vol. 76, no. 260, pp. 1743-1769, 2007. 